\title{
Fast online corrections of tripping responses
}

Zrinka Potocanac ${ }^{1}$, Janneke de Bruin ${ }^{2}$, Susanne van der Veen ${ }^{2}$, Sabine Verschueren ${ }^{3}$, Jaap van Dieën ${ }^{2}$, Jacques Duysens $^{1}$, Mirjam Pijnappels ${ }^{2}$

${ }^{1}$ Department of Kinesiology, KU Leuven, Tervuursevest 101 - box 1501, 3001 Leuven, Belgium

${ }^{2}$ MOVE Research Institute Amsterdam, Faculty of Human Movement Sciences, VU University Amsterdam, Van der Boechorststraat 7, 1081 BT Amsterdam, the Netherlands

${ }^{3}$ Department of Rehabilitation Sciences, KU Leuven, Tervuursevest 101 - box 1501, 3001 Leuven, Belgium

Corresponding author:

Mirjam Pijnappels,

MOVE Research Institute Amsterdam, Faculty of Human Movement Sciences, VU University Amsterdam, Van der Boechorststraat 7, 1081 BT Amsterdam, The Netherlands

E-mail: m.pijnappels@vu.nl

Phone: +31205988467 


\begin{abstract}
Tripping over obstacles is one of the main causes of falls. One potential hazard to actually fall when tripped is inadequate foot landing. Adequate landing is required to control the body's angular momentum, while avoiding dangerous surfaces (slippery patch, uneven ground). To avoid such dangers, foot trajectory needs to be controlled by inhibiting and adjusting the initiated recovery foot path during a tripping reaction. We investigated whether such adjustments can be made without jeopardizing balance recovery.

Sixteen healthy young adults (25.1 \pm 3.2 years) walked at their comfortable speed over a walkway equipped with 14 hidden obstacles. Participants were tripped 10 times in between a random number of normal walking trials; 5 trips included a projection of a forbidden zone $(\mathrm{FZ}, 30 \times 50 \mathrm{~cm})$ at the subject's preferred landing position. Participants were instructed to land their recovery foot outside the FZ, if the FZ was presented. Responses were evaluated in terms of foot position and body angular momentum at and following recovery foot landing.

Participants successfully landed their recovery foot outside the FZ in $80 \%$ of trials, using strategies of either shortening their recovery steps $(84 \%)$ or side stepping (16\%). Their performance improved over trials, and some participants switched strategies. Angular momenta of the adjusted steps remained small at and following recovery foot landing.

Young adults can quickly change foot trajectory after tripping by using different strategies and without detrimental consequences on balance recovery, in terms of the angular momentum. These results open possibilities for training of tripping reactions.
\end{abstract}

Keywords

Gait perturbation, stability, accidental falls, motor inhibition, obstacle avoidance, angular momentum 
Introduction

Movement inhibition is commonly seen in human behavior (Coxon et al. 2009; Verbruggen and Logan 2008) and is reflected in fast online corrections and movement adjustments in response to external perturbations (Desmurget et al. 1999; Oostwoud Wijdenes et al. 2011; Prablanc and Martin 1992). Previous work in this field focused mostly on adjustments of arm movements towards a target (Desmurget et al. 1999; Gosselin-Kessiby et al. 2009; Prablanc and Martin 1992; Shadmehr et al. 2010) using perturbations such as target shifts (Oostwoud Wijdenes et al. 2011; Prablanc and Martin 1992), and visual and proprioceptive feedback manipulations (Goodbody and Wolpert 1998; Körding et al. 2004; Shadmehr et al. 2010). However, very little is known about fast corrections during leg movements, such as gait, although these are very important for successful ambulation. Some attempts were made to study online adjustments of leg movements, mostly during obstacle avoidance (Moraes et al. 2004; Patla et al. 1991; Potocanac et al. 2014; Weerdesteyn et al. 2005a) and step initiation (Tseng et al. 2009). Yet little is known with respect to alternate foot placement after tripping. This is an important issue however, since the occurrence of a fall can be linked to improper placement of the foot after tripping (Pavol et al. 2001; Pijnappels et al. 2005a). In daily life, this proper placement can be difficult in case one has to avoid stepping on "risky" surfaces, like uneven ground or a rainy patch. If fast online adjustments of tripping reactions are possible, this would open possibilities for training and fall prevention in the growing population of older adults (Bieryla et al. 2007; Grabiner et al. 2012; Rosenblatt et al. 2013).

Tripping over obstacles has been shown to be one of the dominant causes of falls in older adults (Berg et al. 1997; Overstall et al. 1977; Rao 2005; Robinovitch et al. 2013) and mechanisms of tripping have been widely explored (Eng et al. 1994; Forner-Cordero et al. 2003; Grabiner et al. 1993; Pavol et al. 2001; Pijnappels et al. 2005b; Pijnappels et al. 2004; Rhea and Rietdyk 2011; Schillings et al. 2000; Schillings et al. 1996; Senden et al. 2014). In general, following a trip, flexion or extension movements of the tripped foot are observed (Eng et al. 1994) and switching between these two types of responses has been reported in some individuals (Forner-Cordero et al. 2005; Forner-Cordero et al. 2003; Schillings et al. 2000), suggesting that online adjustments of tripping responses might be possible, probably in order to reduce the risk of falling. An adequate response to a tripping perturbation is needed to reduce the forward angular momentum that the body obtains from impact with the obstacle (van Dieën et al. 2005; Forner-Cordero 2003; Grabiner et al. 1993). Balance can be recovered after tripping by control of trunk movement (Grabiner et al. 1993; Pavol et al. 2001), generating rapid forces in the support leg during push-off (Pijnappels et al. 2005b; Pijnappels et al. 2004), and proper placement of the recovery foot (Forner-Cordero 2003; Pavol et al. 2001; Schillings et al. 2005). The latter seems especially important as placing the recovery foot anterior to the center of mass $(\mathrm{COM})$ can generate a moment that counteracts the body's forward rotation (Forner-Cordero 2003; Grabiner et al. 1993; Pavol et al. 2001; Schillings et al. 2005). Furthermore, this ability is often impaired in older adults, and especially in older fallers, who have been shown to generate less adequate push-off forces when tripped in comparison with healthy young adults. These inadequate push-off forces result in short recovery steps that insufficiently reduce the body's angular momentum, thus increasing the chance of falling (Pijnappels et al. 2005a). Adjustments of recovery step length might thus be beneficial to avoid falling, but whether or not changes to foot positioning after a trip are possible is not known, as none of the aforementioned studies constrained foot positioning 
after tripping. If these changes are indeed possible foot positioning after perturbations might be trainable, for example by teaching subjects how to improve their tripping response or use safe methods for falling if recovery proves impossible (Weerdesteyn et al. 2006).

The main aim of our study was to establish whether young adults can adjust their tripping responses and whether this affects balance recovery. In order to do so, we assessed whether people can alter their preferred foot landing position in response to a visual forbidden landing zone (FZ) presented at trip onset. The FZ represents a virtual obstacle that needs to be avoided during trip recovery. Based on previous reports on (incidental) step trajectory adjustments during obstacle avoidance (Moraes et al. 2007; Patla et al. 1991) and tripping recovery (Forner-Cordero et al. 2005; FornerCordero et al. 2003; Schillings et al. 2000), we hypothesized that our subjects would be able to adjust their recovery foot landing position when a FZ is presented. Our secondary aim was to evaluate the step strategies and consequences of step adjustments for balance recovery in terms of angular momentum.

\section{Methods}

Sixteen healthy young adults (age $25.1 \pm 3.2$ years, height $178.4 \pm 8.8 \mathrm{~cm}$, weight $73.2 \pm 12.9 \mathrm{~kg}, 6$ females) participated in this study after signing informed consent. Subjects had no walking problems, normal or corrected to normal vision and were able to understand the instructions. The study was in accordance with the guidelines of good clinical practice and approved by the local ethical committee. All subjects gave their informed consent prior to participating in the study.

\section{Experimental setup}

Subjects walked at their comfortable speed over a walkway ( $2.5 \mathrm{~m}$ wide and $12 \mathrm{~m}$ long), equipped with a force plate and 14 obstacles ( $15 \mathrm{~cm}$ high) hidden over a length of one meter (Fig. 1). Any of these obstacles could be released from the floor causing the subject to trip. The obstacle to be released was selected online by an algorithm based on the subjects' kinematic parameters during obstacle approach. More specifically, upon landing of the subject's left foot in the area next to the obstacles, the obstacle closest to the midline of the left foot was selected and triggered to be released to cause a trip at mid-swing of the right leg. This impact at mid swing elicited an elevating strategy, meaning that subjects made a recovery step with the foot that hit the obstacle by lifting it over the obstacle (Eng et al. 1994). We mixed ten tripping trials with a number (3 - 15) of normal walking trials so that the subjects never knew whether or not they would be tripped in that specific trial. Normal walking trials in between tripping trials ensured that the subjects regained their normal walking pattern (Pijnappels et al. 2001) and no significant changes in normal gait parameters were observed after the first trip. Participants were encouraged to maintain their preferred velocity as shown at the start of the experiment. To check for possible anticipatory gait changes during the tripping trials, we calculated walking speed for the stride prior to obstacle contact (see below) and toe clearance as vertical position of the 2nd toe tip at obstacle contact. 
Subjects wore a safety harness attached to a ceiling-mounted rail, protecting them from falling in case they were not able to regain balance after a trip. The safety ropes provided enough slack for unrestrained motion, and a spring, in series with the ropes, ensured smooth restraint in case of a fall. The tripping setup was described in more detail by Pijnappels and co-workers (Pijnappels et al. 2004).

The experiment consisted of two tripping conditions: 'normal tripping' trials and tripping with a presentation of a FZ (T-FZ). The FZ was a $30 \mathrm{~cm}$ wide and $50 \mathrm{~cm}$ long rectangle projected onto the floor by a generic projector. The $\mathrm{FZ}$ size covered the group variability of the recovery foot landing positions of 10 subjects from previous experiments (Pijnappels et al. 2005a; Pijnappels et al. 2005b; Pijnappels et al. 2004). The FZ was triggered $100 \mathrm{~ms}$ prior to the trigger for obstacle release and was individually positioned at the participant's average recovery foot landing position during normal tripping.

Following familiarization with the setup and the safety harness, three normal tripping trials were performed, with the subjects instructed to regain balance in any way that comes naturally. Average recovery foot landing position relative to the obstacle was calculated based on the kinematic data of the foot and used to position the center of the FZ for each participant individually. For the following trials, the subjects were instructed to regain their balance in case of a trip, but to avoid stepping into the FZ if present. Seven more trips (5 T-FZ and 2 'normal' trips) and five trials that included only the FZ without a trip were performed in a pseudorandom manner. The latter condition was used to prevent subjects from associating the $\mathrm{FZ}$ with tripping and was not analyzed.

Full body kinematic data were collected at a sample rate of 50 samples/s using an Optotrak system (Northern Digital Inc., Waterloo, Ont., Canada) consisting of a $4 \times 3$ camera array. Following anthropometric measurements, 12 clusters of three infrared LED's (Light Emitting Diodes) were attached to the body segments (lower arms, upper arms, lower legs, upper legs, feet, trunk, and pelvis) and a pointer was used to indicate 36 anatomical landmarks. This allowed for reconstruction of the subject's body using a 3D full body kinematic model (Kingma et al. 1996). Kinetic data were collected using a custom-made strain gauge force plate of $1 \times 1 \mathrm{~m}$ (sample rate of 200 samples/s), embedded into the walkway in the area where the recovery foot landed.

Data analysis

Ten tripping trials (of four subjects) were excluded from the analysis due to recovery steps using the left foot. Avoiding the $\mathrm{FZ}$ was not a challenge in these trials since the $\mathrm{FZ}$ was positioned based on the assumption that the right foot would be elevated for the recovery step. The two normal trips mixed with the T-FZ trials served as 'catch' trials and were treated as a separate group in the analysis, as these could have been influenced by the FZ trials. Five T-FZ trials were compared to the first three normal trips ( $\mathrm{T}$ trials) and the two 'catch' trip trials (TC trials). Kinematic data were filtered using a $2^{\text {nd }}$ order zero lag Butterworth filter with a cutoff frequency of $5 \mathrm{~Hz}$.

Walking speed was calculated from the displacement of the COM in the stride (defined as the time between two heel strikes of the left foot) prior to obstacle contact. Then, we calculated the spatial and temporal parameters of the recovery step. The timing of subject's contact with the obstacle was determined as the local minimum of foot 
acceleration in the walking direction. The force plate was unloaded prior to the recovery step, so the time of recovery foot landing was identified as the onset of a sudden increase in the vertical force. Manual correction of the recovery foot landing time was needed for one subject, as he performed two steps onto the force plate. Recovery step duration was defined as the time between obstacle contact and recovery step landing. Foot position at landing was determined from the kinematic data as the virtual line connecting the calcaneus and the tip of the second toe. If this line fell fully outside of the FZ, the avoidance was considered successful. In case of a successful avoidance of the FZ, the step was classified into one of the following strategies: step lengthening, step shortening or side stepping (Chen et al. 1994) based on the position of the foot at landing (Fig. 2). The absolute Euclidean distance between the FZ center and center of the line representing the foot was calculated.

Finally, to quantify the effects of adjustments on balance recovery, angular momentum (ANGMOM) of the body around the COM was calculated using a 3D full body kinematic model (Kingma et al. 1996), normalized to bodyweight and quantified at trip onset, landing and $0.3 \mathrm{~s}$ after landing. Additionally, to get insight into the amount of rotation during the recovery step and after landing we calculated the areas under the angular momentum curve from obstacle contact until landing (AUC-CL) and from landing until $0.3 \mathrm{~s}$ after landing (AUC-AL).

Data processing was performed using MATLAB $2011 \mathrm{~b}$ (Mathworks, Natick, MA, USA).

Statistical analysis

Average step duration, absolute distance between the center of the foot and center of the FZ, toe clearance at obstacle contact, and walking speed were compared between T, TC, and T-FZ conditions using paired samples t-tests.

Generalized estimating equations (GEE) were used for analysis of success rates, strategy choices, and angular momenta. GEE is a robust method of estimating the parameters of a generalized linear model with a possible unknown correlation between outcomes, used for estimating the average response over the population. The potential effect of learning on success of FZ avoidance was analyzed using a GEE model with trial ordinal number as a predictor and success as a dichotomous response. The same was done for walking speed as a predictor of success. As different strategies in FZ avoidance were observed, GEE models were used to evaluate effects of body height, walking speed, ANGMOM at trip onset, and trial order on strategy choice. Pearson's correlations were calculated between body height and FZ placement. The ANGMOM at trip onset, recovery step landing and $0.3 \mathrm{~s}$ after landing and areas under the angular momentum curve from obstacle contact until landing (AUC-CL) and from landing until $0.3 \mathrm{~s}$ after landing (AUC-AL) were analyzed using GEE models with five trip strategies (T trials, TC trials, T-FZ step shortening, T-FZ side stepping, and failed T-FZ trials) as a factor and trial ordinal number and walking speed as covariates. This enabled a comparison between the different strategies, while accounting for possible learning effect and differences in walking speed. Step duration was used as an additional covariate for AUC analyses.

All analyses were performed using SPSS Statistics 20 (IBM, Chicago, IL, USA), with a level of statistical significance set to $\alpha=0.05$. 
Results

Mean walking speed in the stride preceding the trip during the T-FZ trials $(1.32 \pm 0.10 \mathrm{~m} / \mathrm{s}, \mathrm{p}=0.005)$ and TC trials $(1.33 \pm 0.09 \mathrm{~m} / \mathrm{s}, \mathrm{p}=0.013)$ was slightly but significantly lower than the speed during the $\mathrm{T}$ trials $(1.40 \pm 0.11 \mathrm{~m} / \mathrm{s})$. Although toe clearance at obstacle contact slightly increased from $0.04 \mathrm{~m}$ during the $\mathrm{T}$ trials to $0.07 \mathrm{~m}$ during the TC and T-FZ trials (both $\mathrm{p}<0.001$ ), trips were successfully elicited given the height of the obstacle of $0.15 \mathrm{~m}$.

Overall, subjects were able to adjust their steps and avoid stepping into the FZ. Subjects failed in 14 (20\%) and succeeded in 57 (80\%) of the T-FZ trials (Fig. 3). The adjusted steps, irrespective of being successful or not, lasted shorter than the first three normal trips ( $T$ trials: $0.48 \pm 0.03 \mathrm{~s}$, T-FZ trials: $0.43 \pm 0.03 \mathrm{~s}, \mathrm{p}=0.002$, Fig. $4 \mathrm{a}$ ) and had a larger distance from the center of the foot to the center of the FZ ( $\mathrm{T}$ trials: $0.10 \pm 0.03 \mathrm{~m}, \mathrm{~T}-\mathrm{FZ}$ trials: $0.43 \pm 0.12 \mathrm{~m}, \mathrm{p}<0.001$, Fig. $4 \mathrm{~b}$ ). Recovery steps in the last two normal 'catch' trips (TC trials) also differed from the first three normal trips ( $\mathrm{T}$ trials): they were of shorter duration $(0.45 \pm 0.03 \mathrm{~s}, \mathrm{p}=0.003)$ and had a larger distance from the center of the foot to the center of the FZ $(0.29 \pm 0.16 \mathrm{~m}, \mathrm{p}<0.001)$. However, this distance was significantly smaller than for the T-FZ steps $(\mathrm{p}<0.001)$, although the TC and T-FZ steps did not differ in duration.

Performance on the T-FZ trials significantly improved over trials, from $60 \%$ of successful FZ avoidances in the first, $73 \%$ in the second, $80 \%$ in the third, $93 \%$ in the fourth and $100 \%$ success rate in the final, fifth trial $(\mathrm{p}<0.001$, Fig. 4c). The GEE model revealed no effect of walking speed on success rate.

\section{Strategies}

Different strategies were used for successful step adjustments. Out of the 57 successful T-FZ trials, step shortening was observed in $48(84 \%)$ trials and side steps (all in lateral direction) were observed in 9 (16\%) trials. Long steps (over the FZ) were not observed. Strategy choice was not associated with trial number, walking speed, or ANGMOM at trip onset, but it was significantly associated with subject's body height (GEE $p=0.035$, exp $(\beta)=0.858)$, meaning that a side step was $14 \%$ less likely to occur with each additional $\mathrm{cm}$ in body height. This can be explained by the fact that the FZ was positioned closer to the tripping obstacle for shorter subjects, based on their absolute shorter normal recovery steps during the $\mathrm{T}$ trials, as evident from the correlation between the subjects' body height and the $\mathrm{FZ}$ position $($ Pearson $\mathrm{r}=0.623, \mathrm{p}=0.010)$.

Angular momentum

Typical examples of ANGMOM curves in all three planes are shown for a T trial (Fig. 5a) and T-FZ (step shortening, Fig. 5b). Rotation in the horizontal plane was minor so the analysis focused on frontal and sagittal planes. Average amounts of ANGMOM, AUC-CL and AUC-AL are shown in Table 1. Group angular momentum data are shown in Fig. 6 and it can be seen that the magnitude of changes in the angular momentum was small. 
Although most subjects preferred step shortening, this type of strategy did not severely affect the angular momentum compared to normal trips; especially in the sagittal plane, were largest effects were expected for step shortening, only small differences in angular momenta were observed.

According to the GEE model, the average ANGMOM at obstacle contact was associated in the frontal plane with strategy $(p=0.003)$ and in the sagittal plane with covariates walking speed $(p=0.003)$ and trial ordinal number $(p=0.046)$. Average amounts of ANGMOM at landing were associated with walking speed $(p=0.006)$ and strategy $(\mathrm{p}=0.049)$ in the frontal plane. ANGMOM $0.3 \mathrm{~s}$ after landing was associated in the frontal plane with strategy $(p=0.006)$ and in the sagittal plane with strategy $(p=0.040)$ and trial ordinal number $(p=0.038)$. Note that both at and $0.3 \mathrm{~s}$ after landing, the average differences in magnitudes of ANGMOM were small for all strategies used.

AUC-CL in the frontal plane was associated with trial ordinal number $(\mathrm{p}=0.001)$, walking speed $(\mathrm{p}=0.048)$, and strategy $(p=0.049)$. In the sagittal plane AUC-CL was associated with step duration $(p<0.001)$ and strategy $(\mathrm{p}=0.007)$. In the $0.3 \mathrm{~s}$ following recovery step landing AUC-AL and strategy were associated in the frontal plane $(p<0.001)$ and borderline significantly associated in the sagittal plane $(p=0.054)$.

\section{Discussion}

We investigated whether and how young adults can adjust their tripping responses and secondly, we explored the consequences of adjustments on balance recovery in terms of angular momentum. As hypothesized, subjects were able to adjust their tripping responses and avoid landing in the FZ. Although individual differences in success rates were present, all subjects succeeded at least once. In their attempts, either successful or unsuccessful with respect to avoiding the FZ, none of our subjects lost balance, and only small changes in the magnitudes of angular momenta were observed.

Adjustment strategies and their consequences

We have shown the ability of young adults to execute online adjustments of their trip recovery step trajectories in response to a visual cue (FZ) in the environment. These findings are in line with previous work on obstacle avoidance, showing the ability to execute two stage corrections of foot placement when a second obstacle is presented in the landing area of the initial obstacle crossing step (Patla et al. 1991). Furthermore, our results confirm incidental reports of individuals changing response step trajectories based on environmental constraints during obstacle avoidance (Moraes et al. 2007) and tripping recovery (Forner-Cordero et al. 2005; Forner-Cordero et al. 2003; Schillings et al. 2000).

Our FZ can be considered a virtual obstacle appearing in one's path following a trip. The FZ approach is similar to that taken in the obstacle avoidance literature by Moraes and co-workers (Moraes et al. 2007; Moraes and Patla 2006) and by Chen and co-workers (Chen et al. 1996; Chen et al. 1994), who investigated avoiding a virtual FZ during normal walking. They showed that FZ avoidance can be achieved by either step lengthening to step over the 
FZ, step shortening to step in front of the FZ, or by stepping to the side (Chen et al. 1994). In our case, to avoid landing into the FZ, one needs to utilize one of these obstacle avoidance strategies, while recovering from a trip. We observed that step shortening was the dominant strategy for FZ avoidance, used in $84.2 \%$ of all successful FZ avoidance trials. This finding is surprising at first sight, as we expected step shortening to be less beneficial in terms of time and angular momentum.

Firstly, as established in obstacle avoidance experiments, step lengthening would allow for more time to implement a step adjustment than shortening. In obstacle avoidance, this time is termed available response time (ART) and defined as the time between obstacle appearance and the time of the foot's collision with the obstacle if there is no avoidance reaction. In our experiment the FZ is a virtual obstacle and the tripping recovery step is in fact an obstacle avoidance step. Hence, because the virtual obstacle (FZ) is presented at trip onset and collision with the FZ would occur at recovery step landing if no adjustment is made, the duration of a 'normal tripping' recovery step (i.e., $\mathrm{T}$ trials) is equivalent to ART. Research on obstacle avoidance in young adults (Chen et al. 1994; Weerdesteyn et al. 2005 b) reported a switch from using predominantly step shortening to lengthening when time available to respond (ART) increases above 250-300 ms. In our case, timing does not seem to be a likely explanation for the occurrence of step shortening: the average durations of both the T steps and T-FZ steps were in the range where young adults prefer step lengthening during obstacle avoidance (T trials $476 \mathrm{~ms}$, T-FZ trials $434 \mathrm{~ms}$ ).

Secondly, in terms of angular momentum, long recovery steps are beneficial for reducing the amount of angular momentum generated by the trip in the sagittal plane (Forner-Cordero et al. 2003; Grabiner et al. 1993; Pavol et al. 2001; Pijnappels et al. 2005a; Schillings et al. 2005), and step shortening could be potentially destabilizing as it could result in increased angular momentum at landing (Pavol et al. 2001; Pijnappels et al. 2005a; Weerdesteyn et al. 2005b). In our experiment, only minor effects of step shortening were seen on angular momentum compared to normal tripping and our subjects were able to regain balance, as indicated by the small magnitudes of angular momenta in both sagittal and frontal planes at and following recovery step landing.

The main reason why our subjects preferred step shortening was likely the size of the FZ $(30 \mathrm{~cm}$ wide and $50 \mathrm{~cm}$ long). We selected a FZ large enough to cover the group variability in the landing foot positions obtained from previous tripping experiments (Pijnappels et al. 2005a; Pijnappels et al. 2005b; Pijnappels et al. 2004), to ensure that successful T-FZ trials were the result of recovery step adjustments and not chance. Probably, the FZ was positioned at such a distance and was so long, that it was physically too challenging for subjects to avoid it by further lengthening their steps. Namely, on average, the far edge of the FZ was located at $1.28 \mathrm{~m}$ from the obstacle. In comparison, the average step length preceding the trip was $0.75 \mathrm{~m}$. This means that a successful crossing of the FZ with a long step would require a lengthening of the step by about $0.52 \mathrm{~m}$ (i.e., $70 \%$ ).

Our subjects used two strategies for successful FZ avoidance (step shortening and side stepping). Strategy selection was related to body height; taller subjects mostly used step shortening, while shorter subjects more often used side steps. This effect of body height on strategy choice may have been caused by the relative distance between the obstacle and the FZ. Shorter subjects made shorter recovery steps during the T trials and therefore their FZ was 
positioned closer to the tripping obstacle. Hence, these subjects probably preferred side stepping because the limited space between the obstacle and FZ would make step shortening too challenging for them. However, based on the magnitudes of angular momenta of the adjusted steps, we believe that recovery step adjustments (irrespective of the strategy used) did not threaten balance recovery following a trip. Our reasoning is based on the fact that, although strategy was significantly associated with angular momentum, the magnitudes of the measured angular momenta were small compared to previously reported data of successful trip recoveries in young adults. In our experiment the largest changes in angular momentum between trials with and without a FZ were seen at landing for step shortening in the frontal plane and for failed T-FZ trials in the sagittal plane. However, the angular momenta of these adjusted steps were still smaller than previously reported values for successful trip recovery in young adults using the same setup (Pijnappels et al. 2010; Pijnappels et al. 2005a), both in the sagittal plane $\left(>0.1 \mathrm{~m}^{2} \mathrm{~s}^{-1}\right.$; in our experiment $\left.0.06 \mathrm{~m}^{2} \mathrm{~s}^{-1}\right)$ and in the frontal plane $\left(-2.99 \mathrm{~m}^{2} \mathrm{~s}^{-1}\right.$; in our experiment $\left.0.05 \mathrm{~m}^{2} \mathrm{~s}^{-1}\right)$. Therefore we believe that these magnitudes of angular momenta do not present a problem for healthy young subjects to successfully recover from the trip, as was the case in our experiment where none of the subjects fell.

Learning effect and its implications

Although each subject was able to adjust recovery steps, not all of our subjects were successful in avoiding the FZ in all of the trials. However, they all improved performance with practice, and were able to avoid the FZ in at least one trial. Success rates significantly improved over trials, from $60 \%$ of successful $\mathrm{FZ}$ avoidances in the first, to a $100 \%$ success rate in the final, fifth T-FZ trial.

Learning effects were also reflected in the two normal 'catch' trips in between the T-FZ trials. These trips differed from the first three normal trips in walking speed, toe clearance at obstacle contact, and recovery step duration. Most importantly, the average distance from the center of the foot to the center of the FZ increased by $0.19 \mathrm{~m}$ from the first three normal trips. Landing at a more remote position from the center of the FZ is the appropriate strategy to assist avoiding a FZ in case it would appear and indeed, if the FZ had been presented in these trials, our subjects would have avoided it successfully using step shortening in 11 (out of 31) trials. This suggests that anticipation affected the tripping response even if there was no FZ and illustrates that learning occurred, in line with previous work using similar tripping responses (Pijnappels et al. 2006).

Our data are in line with several studies that reported anticipation and learning effects following trips and slips. For example, following a contact of the trailing limb with an obstacle, subjects increased their trail limb toe clearance and peak toe elevation (Rhea and Rietdyk 2011). In another study (Wang et al. 2012), after being exposed to eight consecutive overground trips, young adults exhibited changes in their kinematic parameters both in anticipation of the trip (reduced COM velocity and forward instability and increased toe clearance) and during trip recovery (decreased maximal trunk flexion, increased hip height, posterior shift in the COM position and decreased COM velocity). These adjustments were beneficial for reducing angular momentum and regaining balance, but were based on trip anticipation and not on applying a new strategy or responding to environmental constraints. The same group (Pai et al. 2010) reported improvements in 'fall-resisting skills' when young adults were exposed to blocks of 
consecutive trips and slips. However, a major limitation of these experiments was a decrease in walking speed observed prior to the trip of interest, which was not controlled for in the data analysis. This limits the conclusions that could be drawn, as walking speed at impact largely influences success of tripping recovery (van Dieën et al. 2005; Pavol et al. 2001). Several other groups have reported improvements in tripping responses following a number of actual (Pijnappels et al. 2005a) or simulated trips (Bieryla et al. 2007). Overall, these findings seem to indicate a possibility for learning to improve tripping responses. While a strong limitation here is that in everyday life it is impossible to continuously anticipate a possible trip or slip, this should not discourage future training of older adults.

Older subjects have frequently been shown to make recovery steps that are too short for successful balance recovery (Pijnappels et al. 2005a). Therefore, if older adults are also able to adjust and improve their tripping responses such that they lengthen their recovery steps, this might potentially reduce the number of falls. The feasibility of such an approach is supported by the changes in the kinematics of tripping responses of healthy older adults following a training consisting of simulated slips on a treadmill (Bieryla et al. 2007) and by the ability of older adults to increase their step length following five anticipated trips simulated by treadmill accelerations from quiet standing (Owings et al. 2001). Training consisting of such treadmill accelerations was shown to decrease the number of falls (Grabiner et al. 2012; Rosenblatt et al. 2013). However, in our experiment young adults did not use step lengthening to avoid landing in the FZ, probably due to its size and position. Whether or not older adults would be able to adjust their tripping responses in order to avoid landing in the FZ, and whether they would be able to lengthen their recovery steps to do so, needs further investigation.

\section{Conclusion}

Our study showed that young adults are able to execute fast online adjustments of trip recovery steps in order to avoid landing on a FZ and that they do so by using different strategies. The adjustments (irrespective of strategies) do not seem to threaten balance recovery, in terms of the angular momentum at, and following, recovery foot landing. Subjects improved their success rates over trials, could switch between strategies, and adjusted their responses even when no FZ was presented. These behavioral results serve as a basis for further research on the underlying mechanisms for inhibition and adjustments during tripping and on the feasibility to include trip recovery training for fall prevention in older adults.

\section{Acknowledgements}

We would like to thankfully acknowledge the help of Sjoerd Bruijn and Gert Faber in using the 3D kinematic model and Richard Casius and Leon Schutte for technical help. This research was funded by the European Commission through MOVE-AGE, an Erasmus Mundus Joint Doctorate program (2011-0015). JD has been funded by F.W.O. grant (G.0901.11) and by the Interuniversity Attraction Poles Program initiated by the Belgian Science Policy Office (P7/11). 
References

Berg W, Alessio H, Mills E, Tong C (1997) Circumstances and consequences of falls in independent communitydwelling older adults. Age Ageing 26:261-268.

Bieryla KA, Madigan ML, Nussbaum MA (2007) Practicing recovery from a simulated trip improves recovery kinematics after an actual trip. Gait Posture 26:208-13.

Chen HC, Ashton-Miller JA, Alexander NB, Schultz AB (1994) Age effects on strategies used to avoid obstacles. Gait Posture 2:139-146.

Chen HC, Schultz AB, Ashton-Miller JA, Giordani B, Alexander NB, Guire KE (1996) Stepping over obstacles: dividing attention impairs performance of old more than young adults. J Gerontol A Biol Sci Med Sci 51:M116-M122.

Coxon JP, Stinear CM, Byblow WD (2009) Stop and go: the neural basis of selective movement prevention. J Cogn Neurosci 21:1193-1203.

Desmurget M, Epstein CM, Turner RS, Prablanc C, Alexander GE, Grafton ST (1999) Role of the posterior parietal cortex in updating reaching movements to a visual target. Nat Neurosci 2:563-7.

Van Dieën JH, Pijnappels M, Bobbert MF (2005) Age-related intrinsic limitations in preventing a trip and regaining balance after a trip. Saf Sci 43:437-453.

Eng JJ, Winter DA, Patla AE (1994) Strategies for recovery from a trip in early and late swing during human walking. Exp Brain Res 102:339-349.

Forner-Cordero A (2003) Human gait, stumble and... fall? Mechanical Limitations of the Recovery from a Stumble. Twente University, Enschede, The Netherlands

Forner-Cordero A, Koopman HFJM, van der Helm FCT (2003) Multiple-step strategies to recover from stumbling perturbations. Gait Posture 18:47-59.

Forner-Cordero A, Koopman HJFM, van der Helm FCT (2005) Energy analysis of human stumbling: the limitations of recovery. Gait Posture 21:243-54.

Goodbody SJ, Wolpert DM (1998) Temporal and amplitude generalization in motor learning. J Neurophysiol 79:1825-38.

Gosselin-Kessiby N, Kalaska JF, Messier J (2009) Evidence for a proprioception-based rapid on-line error correction mechanism for hand orientation during reaching movements in blind subjects. J Neurosci 29:3485-96.

Grabiner M, Koh T, Lundin T, Jahnigen D (1993) Kinematics of recovery from a stumble. J Gerontol Med Sci 48:M97-M102.

Grabiner MD, Bareither M Lou, Gatts S, Marone J, Troy KL (2012) Task-specific training reduces trip-related fall risk in women. Med Sci Sports Exerc 44:2410-4.

Kingma I, de Looze MP, Toussaint HM, Klijnsma HG, Bruijnen TBM (1996) Validation of a full body 3-D dynamic linked segment model. Hum Mov Sci 15:833-860.

Körding KP, Ku S, Wolpert DM (2004) Bayesian integration in force estimation. J Neurophysiol 92:3161-5. 
Moraes R, Allard F, Patla AE (2007) Validating determinants for an alternate foot placement selection algorithm during human locomotion in cluttered terrain. J Neurophysiol 98:1928-40.

Moraes R, Lewis MA, Patla AE (2004) Strategies and determinants for selection of alternate foot placement during human locomotion: influence of spatial and temporal constraints. Exp Brain Res 159:1-13.

Moraes R, Patla AE (2006) Determinants guiding alternate foot placement selection and the behavioral responses are similar when avoiding a real or a virtual obstacle. Exp Brain Res 171:497-510.

Oostwoud Wijdenes L, Brenner E, Smeets JBJ (2011) Fast and fine-tuned corrections when the target of a hand movement is displaced. Exp Brain Res 214:453-62.

Overstall P, Exton-Smith A, Imms F, Johnson A (1977) Falls in the elderly related to postural imbalance. Br Med J $1: 261-264$.

Owings TM, Pavol MJ, Grabiner MD (2001) Mechanisms of failed recovery following postural perturbations on a motorized treadmill mimic those associated with an actual forward trip. Clin Biomech 16:813-9.

Pai Y-C, Bhatt T, Wang E, Espy D, Pavol MJ (2010) Inoculation against falls: rapid adaptation by young and older adults to slips during daily activities. Arch Phys Med Rehabil 91:452-9.

Patla AE, Beuter A, Prentice S (1991) A two stage correction of limb trajectory to avoid obstacles during stepping. Neurosci Res Commun 8:153-159.

Pavol MJ, Owings TM, Foley KT, Grabiner M (2001) Mechanisms leading to a fall from an induced trip in healthy older adults. J Gerontol A Biol Sci Med Sci 56:M428-37.

Pijnappels M, Bobbert MF, van Dieën JH (2001) Changes in walking pattern caused by the possibility of a tripping reaction. Gait Posture 14:11-18.

Pijnappels M, Bobbert MF, van Dieën JH (2005a) Push-off reactions in recovery after tripping discriminate young subjects, older non-fallers and older fallers. Gait Posture 21:388-394.

Pijnappels M, Bobbert MF, van Dieën JH (2005b) Control of support limb muscles in recovery after tripping in young and older subjects. Exp Brain Res 160:326-333.

Pijnappels M, Bobbert MF, van Dieën JH (2004) Contribution of the support limb in control of angular momentum after tripping. J Biomech 37:1811-1818.

Pijnappels M, Bobbert MF, van Dieën JH (2006) EMG modulation in anticipation of a possible trip during walking in young and older adults. J Electromyogr Kinesiol 16:137-143.

Pijnappels M, Kingma I, Wezenberg D, Reurink G, van Dieën JH (2010) Armed against falls: the contribution of arm movements to balance recovery after tripping. Exp Brain Res 201:689-699.

Potocanac Z, Hoogkamer W, Carpes FP, Pijnappels M, Verschueren SMP, Duysens J (2014) Response inhibition during avoidance of virtual obstacles while walking. Gait Posture 39:641-4.

Prablanc C, Martin O (1992) Automatic control during hand reaching at undetected two-dimensional target displacements. J Neurophysiol 67:455-69.

Rao SS (2005) Prevention of falls in older patients. Am Fam Physician 72:81-8. 
Rhea CK, Rietdyk S (2011) Influence of an unexpected perturbation on adaptive gait behavior. Gait Posture 34:43941.

Robinovitch SN, Feldman F, Yang Y, Schonnop R, Leung PM, Sarraf T, Sims-Gould J, Loughin M (2013) Video capture of the circumstances of falls in elderly people residing in long-term care: an observational study. Lancet 381:47-54.

Rosenblatt NJ, Marone J, Grabiner MD (2013) Preventing trip-related falls by community-dwelling adults: a prospective study. J Am Geriatr Soc 61:1629-31.

Schillings AM, Mulder T, Duysens J (2005) Stumbling Over Obstacles in Older Adults Compared to Young Adults. J Neurophysiol 94:1158-1168.

Schillings AM, Van Wezel BM, Duysens J (1996) Mechanically induced stumbling during human treadmill walking. J Neurosci Methods 67:11-7.

Schillings AM, van Wezel BM, Mulder T, Duysens J (2000) Muscular responses and movement strategies during stumbling over obstacles. J Neurophysiol 83:2093-102.

Senden R, Savelberg HHCM, Adam J, Grimm B, Heyligers IC, Meijer K (2014) The influence of age, muscle strength and speed of information processing on recovery responses to external perturbations in gait. Gait Posture 39:513-7.

Shadmehr R, Smith MA, Krakauer JW (2010) Error correction, sensory prediction, and adaptation in motor control. Annu Rev Neurosci 33:89-108.

Tseng S-C, Stanhope SJ, Morton SM (2009) Impaired reactive stepping adjustments in older adults. J Gerontol A Biol Sci Med Sci 64:807-815.

Verbruggen F, Logan GD (2008) Response inhibition in the stop-signal paradigm. Trends Cogn Sci 12:418-424.

Wang T-Y, Bhatt T, Yang F, Pai Y-C (2012) Adaptive control reduces trip-induced forward gait instability among young adults. J Biomech 45:1169-75.

Weerdesteyn V, Nienhuis B, Duysens J (2005a) Advancing age progressively affects obstacle avoidance skills in the elderly. Hum Mov Sci 24:865-880.

Weerdesteyn V, Nienhuis B, Mulder T, Duysens J (2005b) Older women strongly prefer stride lengthening to shortening in avoiding obstacles. Exp Brain Res 161:39-46.

Weerdesteyn V, Rijken H, Geurts ACH, Smits-Engelsman BCM, Mulder T, Duysens J (2006) A five-week exercise program can reduce falls and improve obstacle avoidance in the elderly. Gerontology 52:131-141. 
Fig. 1 Experimental setup. Subjects walked at their comfortable speed on a walkway equipped with a force plate and 14 obstacles. The obstacles were embedded in the flooring and could be released causing the subject to trip at mid swing. In 5 out of 10 tripping trials, a forbidden zone (FZ) was projected at trip onset onto the floor at the subject's preferred recovery step landing position, relative to the selected obstacle. Subjects were instructed to regain their balance following a trip, but avoid landing their foot in the FZ if presented

Fig. 2 Strategies used for FZ avoidance. Trials in which the subjects successfully avoided landing their foot into the FZ were classified based on the position of the foot at landing. If the subject used a large step to step over the FZ with the full foot the trial was termed step lengthening, if a short step was made, such that the subject landed their full foot in front of the FZ, the trial was termed a step shortening, and all other successful trials were defined as side stepping. Unsuccessful trials were the trials in which any part of the foot, defined as a virtual line connecting the calcaneus and the tip of the second toe, overlapped with the FZ

Fig. 3 Individual responses to the T-FZ trials. All subjects succeeded in avoiding the FZ at least once, although individual differences in success rates are evident. Note that two subjects changed strategies during the experiment (subjects 2 and 5). One of these can be attributed to the strategy definition used (subject 2) whereas the other (subject 5) reflects a change in behavior. Ten tripping trials (of four subjects) were excluded from the analysis, which left subject 3 without any T-FZ trials. Trials were excluded due to subjects using the left foot for recovery steps, which made avoiding the FZ easy, as it was positioned with an expectation of subjects using an elevating strategy and the right foot

Fig. 4 Average step duration for T, TC, and T-FZ trials (a), distance from the center of the foot to the center of the FZ (b) for T, TC, and T-FZ trials and percentage of successful T-FZ trials per trial ordinal number. Error bars denote standard deviations and * denotes statistical significance $(\mathrm{p}<0.05)$

Fig. 5 Angular momentum around the COM (ANGMOM) normalized to subjects' body weight. Typical ANGMOM curves in the frontal, sagittal and horizontal planes for a 'normal trip' before the first presentation of the FZ (a) and a successful short step avoidance of the FZ (b). Vertical lines denote timings of trip onset, recovery foot landing and $0.3 \mathrm{~s}$ after recovery foot landing

Fig. 6 Angular momentum around the COM (ANGMOM) normalized to subjects' body weight. Group average amounts of ANGMOM at trip onset (a), landing (b) and $0.3 \mathrm{~s}$ after landing (c), and areas under the ANGMOM curve (AUC) from obstacle contact until landing (d) and from landing until $0.3 \mathrm{~s}$ after landing (e). Error bars denote standard errors of the mean and statistically significant effects of strategy are denoted by their $p$ values $(p<0.05)$. Note that the effect of strategy on the sagittal plane AUC $0.3 \mathrm{~s}$ after landing is only borderline significant 
Fig. 1 Experimental setup. Subjects walked at their comfortable speed on a walkway equipped with a force plate and 14 obstacles. The obstacles were embedded in the flooring and could be released causing the subject to trip at mid swing. In 5 out of 10 tripping trials, a forbidden zone (FZ) was projected at trip onset onto the floor at the subject's preferred recovery step landing position, relative to the selected obstacle. Subjects were instructed to regain their stability following a trip, but avoid landing their foot in the FZ if presented 
Fig. 2 Strategies used for FZ avoidance. Trials in which the subjects successfully avoided landing their foot into the FZ were classified based on the position of the foot at landing. If the subject used a large step to step over the FZ with the full foot the trial was termed step lengthening, if a short step was made, such that the subject landed their full foot in front of the FZ, the trial was termed a step shortening, and all other successful trials were defined as side stepping. Unsuccessful trials were the trials in which any part of the foot, defined as a virtual line connecting the calcaneus and the tip of the second toe, overlapped with the FZ $83 \times 55 \mathrm{~mm}(300 \times 300$ DPI) 
Fig. 3 Individual responses to the T-FZ trials. All subjects succeeded in avoiding the FZ at least once, although individual differences in success rates are evident. Note that two subjects changed strategies during the experiment (subjects 2 and 5). One of these can be attributed to the strategy definition used (subject 2) whereas the other (subject 5) reflects a change in behavior. Ten tripping trials (of four subjects) were excluded from the analysis, which left subject 3 without any T-FZ trials. Trials were excluded due to subjects using the left foot for recovery steps, which made avoiding the FZ easy, as it was positioned with an expectation of subjects using an elevating strategy and the right foot $129 \times 203 \mathrm{~mm}(300 \times 300 \mathrm{DPI})$ 

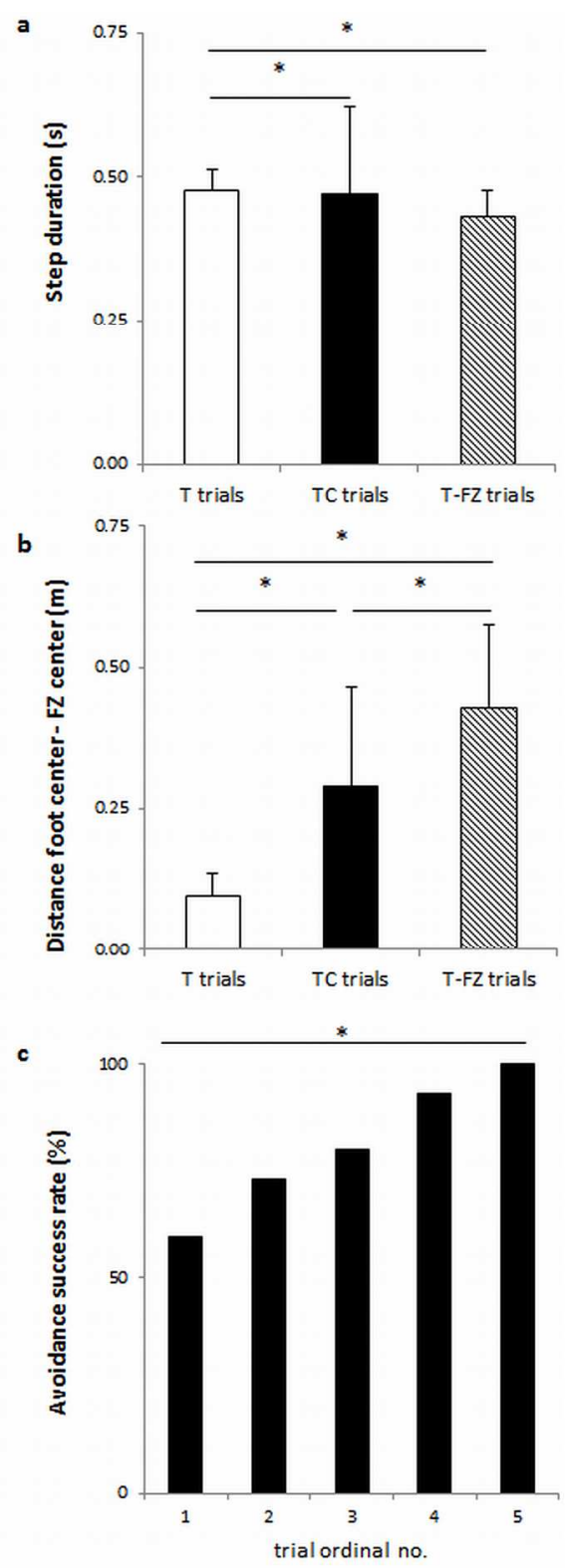

Fig. 4 Average step duration for T, TC, and T-FZ trials (a), distance from the center of the foot to the center of the FZ (b) for T, TC, and T-FZ trials and percentage of successful T-FZ trials per trial ordinal number. Error bars denote standard deviations and * denotes statistical significance $(p<0.05)$ $83 \times 231 \mathrm{~mm}(300 \times 300 \mathrm{DPI})$ 
normal trip

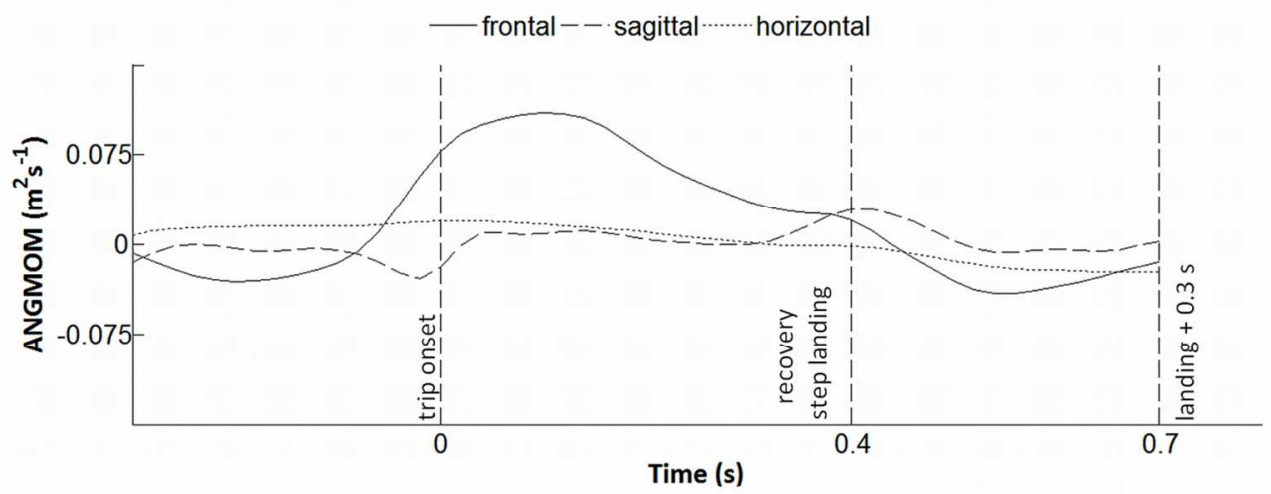

b

trip with a FZ (step shortening)

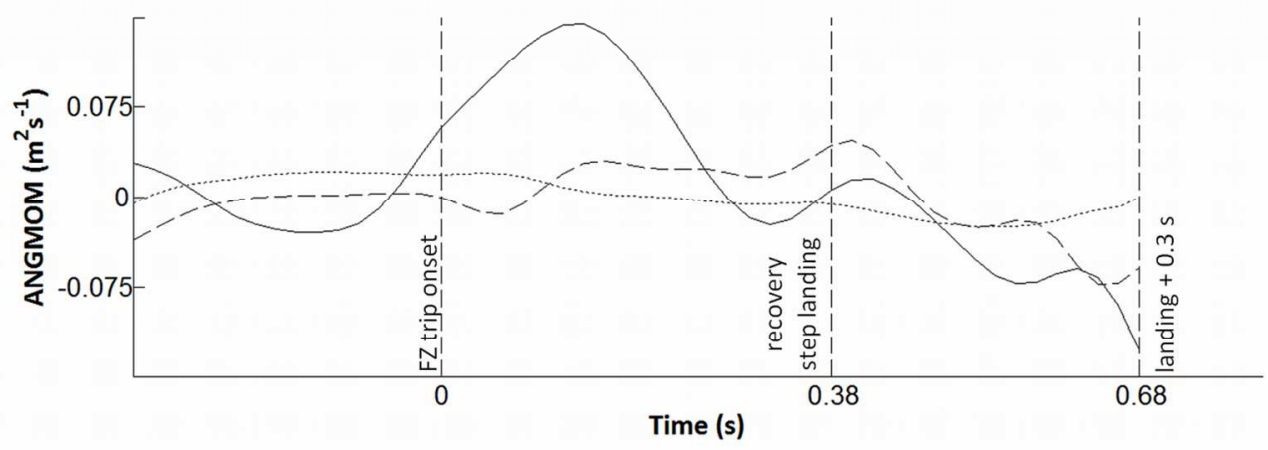

Fig. 5 Angular momentum around the COM (ANGMOM) normalized to subjects' body weight. Typical ANGMOM curves in the frontal, sagittal and horizontal planes for a 'normal trip' (a) and a successful short step avoidance of the FZ (b). Vertical lines denote timings of trip onset, recovery foot landing and $0.3 \mathrm{~s}$ after recovery foot landing $129 \times 124 \mathrm{~mm}(300 \times 300 \mathrm{DPI})$ 
a

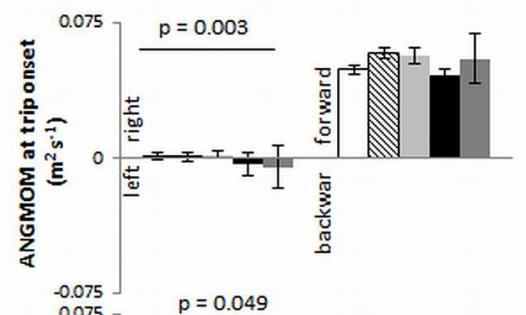

b

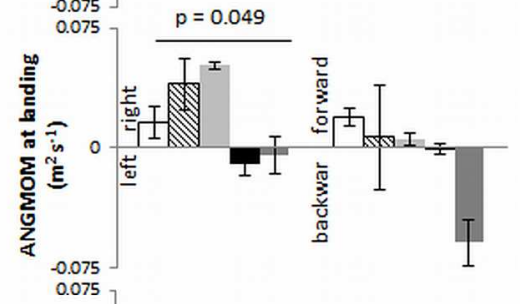

c

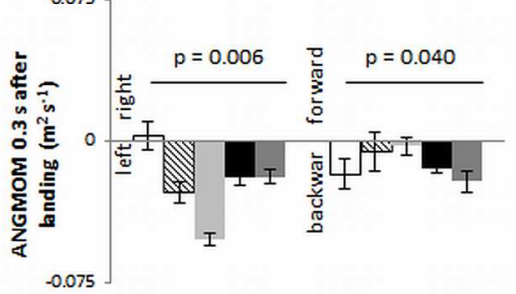

d

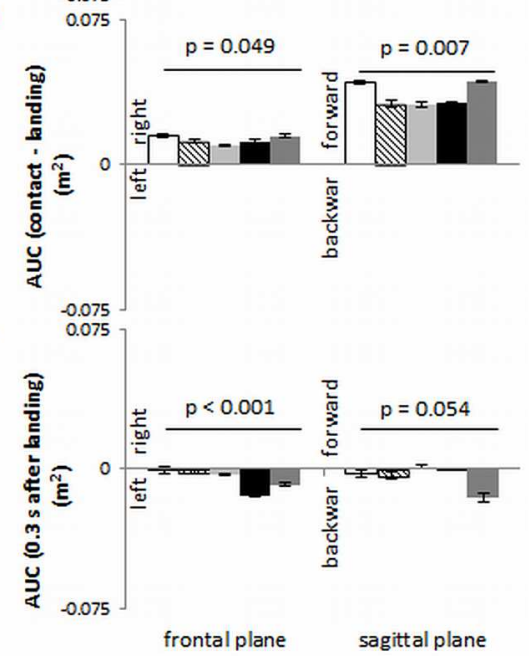

Fig. 6 Angular momentum around the COM (ANGMOM) normalized to subjects' body weight. Group average amounts of ANGMOM at trip onset (a), landing (b) and $0.3 \mathrm{~s}$ after landing (c), and areas under the ANGMOM curve (AUC) from obstacle contact until landing (d) and from landing until $0.3 \mathrm{~s}$ after landing (e). Error bars denote standard errors of the mean and statistically significant effects of strategy are denoted by their $p$ values $(p<0.05)$. Note that the effect of strategy on the sagittal plane AUC $0.3 \mathrm{~s}$ after landing is only borderline significant $83 \times 231 \mathrm{~mm}(300 \times 300 \mathrm{DPI})$ 
Table 1. Average amounts of angular momentum (ANGMOM) at trip onset, recovery step landing and $0.3 \mathrm{~s}$ after recovery step landing and areas under the angular momentum curve from contact to recovery step landing (AUC-CL) and for $0.3 \mathrm{~s}$ after recovery step landing (AUC-AL) in the sagittal and frontal planes. Data are shown for 'normal' tripping trials before the first presentation of the FZ (T trials), 'normal' tripping trials after the first presentation of the FZ (TC trials), successful forbidden zone (FZ) avoidances using a step shortening or side stepping, and unsuccessful FZ avoidances (failed T-FZ trials). Amounts are shown in $\mathrm{m}^{2} \mathrm{~s}^{-1}$ (ANGMOM) and $\mathrm{m}^{2}(\mathrm{AUC})$ as mean $\pm \mathrm{SD}$.

\begin{tabular}{lcccccc}
\hline & & T trials & TC trials & $\begin{array}{c}\text { Step } \\
\text { shortening }\end{array}$ & Side stepping & $\begin{array}{c}\text { Failed T-FZ } \\
\text { trials }\end{array}$ \\
\hline \multirow{2}{*}{ ANGMOM } & frontal & $0.001 \pm 0.014$ & $0.001 \pm 0.014$ & $0.002 \pm 0.014$ & $-0.004 \pm 0.011$ & $-0.005 \pm 0.014$ \\
& sagittal & $0.049 \pm 0.016$ & $0.058 \pm 0.018$ & $0.057 \pm 0.018$ & $0.046 \pm 0.018$ & $0.055 \pm 0.023$ \\
\hline \multirow{2}{*}{ ANGMOM } & & & \multicolumn{7}{c}{ at recovery step landing } \\
& frontal & $0.016 \pm 0.119$ & $0.040 \pm 0.087$ & $0.052 \pm 0.028$ & $-0.010 \pm 0.078$ & $-0.005 \pm 0.145$ \\
\multirow{2}{*}{ AUC-CL } & sagittal & $0.019 \pm 0.072$ & $0.007 \pm 0.181$ & $0.006 \pm 0.052$ & $-0.001 \pm 0.042$ & $-0.059 \pm 0.171$ \\
& frontal & $0.015 \pm 0.014$ & $0.012 \pm 0.005$ & $0.010 \pm 0.006$ & $0.012 \pm 0.009$ & $0.015 \pm 0.010$ \\
& sagittal & $0.042 \pm 0.013$ & $0.031 \pm 0.011$ & $0.031 \pm 0.012$ & $0.031 \pm 0.014$ & $0.043 \pm 0.009$ \\
\hline \multirow{2}{*}{ ANGMOM } & & & 0.3 s after recovery step landing & & \\
& frontal & $0.002 \pm 0.092$ & $-0.028 \pm 0.032$ & $-0.052 \pm 0.040$ & $-0.019 \pm 0.045$ & $-0.019 \pm 0.047$ \\
\multirow{2}{*}{ AUC-AL } & frontal & $-0.018 \pm 0.101$ & $-0.006 \pm 0.057$ & $-0.003 \pm 0.063$ & $-0.015 \pm 0.030$ & $-0.022 \pm 0.069$ \\
& sagittal & $-0.003 \pm 0.026$ & $-0.004 \pm 0.011$ & $0.001 \pm 0.010$ & $-0.001 \pm 0.006$ & $-0.016 \pm 0.029$ \\
\hline
\end{tabular}

